\title{
Ectopic Precarinal Space Parathyroid Carcinoma: A Rare Entity with a Diagnostic Dilemma
}

\author{
Rakesh Bobba ${ }^{1}$, PS Venkatesh Rao ${ }^{2}$, Shilpa Rudradevaru ${ }^{3}$, Ravi S Shetty ${ }^{4}$, Nagamalesh Udigala Madappa ${ }^{5}$, Pramila Kalra ${ }^{6}$
}

\begin{abstract}
Parathyroid carcinomas are rare tumors that arise in the normally located or an ectopic gland. Here, we present a case of a 36-year-old female patient who presented with a history of generalized bone pain, associated with multiple fractures and with elevated serum calcium, parathyroid hormone (PTH) levels, and low vitamin D levels. Biochemical evaluation suggested primary hyperparathyroidism. Initial technetium-99m sestamibi scan and choline 11 scans were misleading and surgical exploration of the neck and superior mediastinum revealed three small parathyroid glands, right superior and inferior, and left superior, all unremarkable on biopsy. Initial surgery failed to alleviate the symptoms. An ectopic parathyroid adenoma was localized in the precarinal space on a gallium 68 DOTANOC PET scan. Repeat surgery by sternotomy, transpericardial approach, and exploration of the mediastinum with intraoperative PTH monitoring was successful in identification and complete excision of parathyroid carcinoma. Operative management of patients with persistent hyperparathyroidism is difficult despite the new advances in radiological imaging. Patients are better managed with a combined approach of biochemical tests, imaging, and intraoperative parathyroid hormone monitoring.

Keywords: DOTANOC, Ectopic adenoma, Ectopic parathyroid carcinoma, Hypercalcemia, Hyperparathyroidism, Intraoperative parathyroid hormone, Precarinal space.

World Journal of Endocrine Surgery (2020): 10.5005/jp-journals-10002-1267
\end{abstract}

\section{INTRODUCTION}

Parathyroid carcinomas are uncommonly seen and the ectopic location of parathyroid carcinoma is very rare. Parathyroid carcinoma is a rare endocrine malignancy, presenting with signs and symptoms of hypercalcemia in the fourth or fifth decade of life. It is clinically indistinguishable from benign cases of hyperparathyroidism, and the pathologic diagnosis is difficult.

These may be sporadic or as part of a genetic syndrome. Ectopic parathyroid carcinoma is an even rarer entity. Parathyroid carcinomas present as diagnostic dilemmas, and there are no specific guidelines on their management. We present a case of parathyroid carcinoma ectopically located in the precarinal space and the difficulties in the diagnosis and management are discussed.

Possible locations of the embryologically ectopic carcinoma are the aortopulmonary window, the deep anterior mediastinum the posterior mediastinum, or within the substance of the thyroid gland. 'Parathyroid adenomas located in the mediastinum, or high in the neck, in or lateral to the cervical neurovascular bundle, or into the thyroid gland are referred to as major ectopy. ${ }^{2}$

\section{Case Description}

A 36-year-old female patient presented with a history of generalized bone pain for the past 8 years, associated with multiple fractures. The patient underwent multiple surgeries for the fractures, but the fractures were nonhealing and disabling. The patient became bedridden due to these fractures. The patient was referred to the Endocrinology Department for further evaluation.

Physical examination was unremarkable other than fractures. Laboratory studies revealed an elevated serum calcium levels and increased PTH levels and low vitamin D levels (corrected serum calcium levels of $14.3 \mathrm{mg} / \mathrm{dL}$, vitamin D level was $14.37 \mathrm{ng} / \mathrm{mL}$, phosphorous, and PTH values were $1.4 \mathrm{mg} / \mathrm{dL}$ and $>1,900 \mathrm{pg} / \mathrm{mL}$, respectively). X-ray showed clear evidence of osteolysis with

\footnotetext{
${ }^{1,6}$ Department of Endocrinology, MS Ramaiah Medical College and Hospital, Bengaluru, Karnataka, India

${ }^{2}$ Department of Surgery, Kadri Clinic, Bengaluru, Karnataka, India

${ }^{3,4}$ Department of Cardiothoracic and Vascular Surgery, Ramaiah Memorial Hospital and Narayana Heart Centre, Bengaluru, Karnataka, India

${ }^{5}$ Department of Cardiology, Ramaiah Memorial Hospital and Narayana Heart Centre, Bengaluru, Karnataka, India

Corresponding Author: Pramila Kalra, Department of Endocrinology, MS Ramaiah Medical College and Hospital, Bengaluru, Karnataka, India, Phone: +91 9972404369, e-mail: kalrapramila@gmail.com

How to cite this article: Bobba R, Rao PSV, Rudradevaru S, et al. Ectopic Precarinal Space Parathyroid Carcinoma: A Rare Entity with a Diagnostic Dilemma. World J Endoc Surg 2020;12(1):23-26.

Source of support: Nil

Conflict of interest: None
}

multiple bone cysts (Fig. 1). The patient has hypercalcemia in spite of vitamin $\mathrm{D}$ deficiency. The diagnosis of primary hyperparathyroidism was made.

To localize the adenoma, the patient was subjected to a technetium-99m sestamibi scan and computerized tomography scan which showed a lesion in the superior mediastinum between the manubrium and the first branch (brachiocephalic artery) of the aorta. The initial surgery was planned through neck approach. Following superior mediastinal and neck exploration surgery (Fig. 2), no parathyroid tumor was found and the patient continued to be symptomatic, with persistently high parathyroid hormone levels and serum calcium levels postoperatively. The patient was then subjected to repeat technetium-99m sestamibi (Fig. 3), choline positron emission tomography (PET) scan (Fig. 4) and multiple site venous sampling. None of these helped identify the tumor correctly. The choline PET scan showed doubtful lesion abutting the anterolateral aspect of 


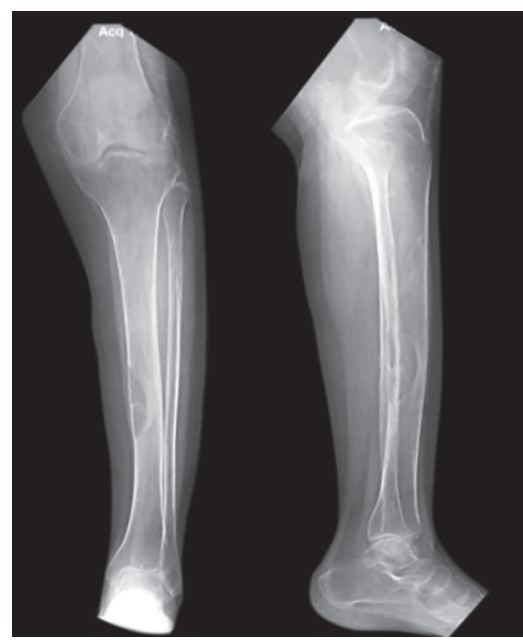

Fig. 1: X-ray leg showing osteolytic lesion

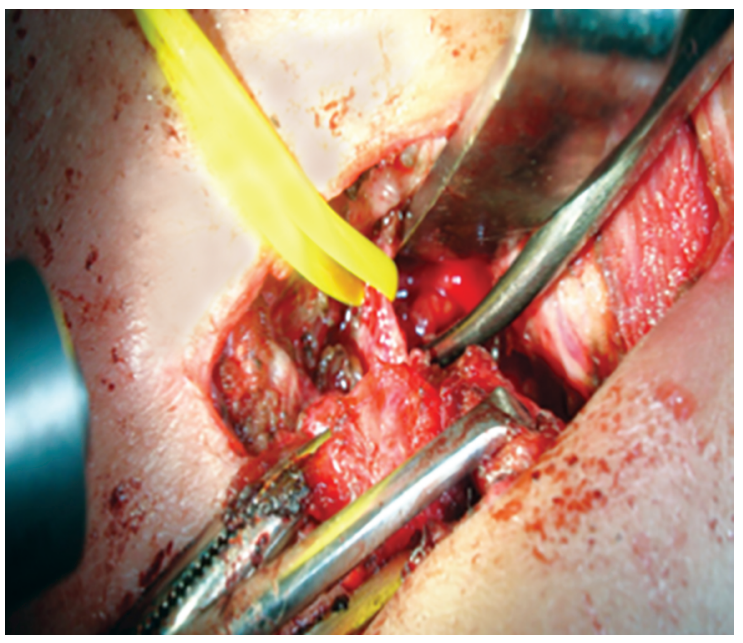

Fig. 2: Operative picture showing neck exploration

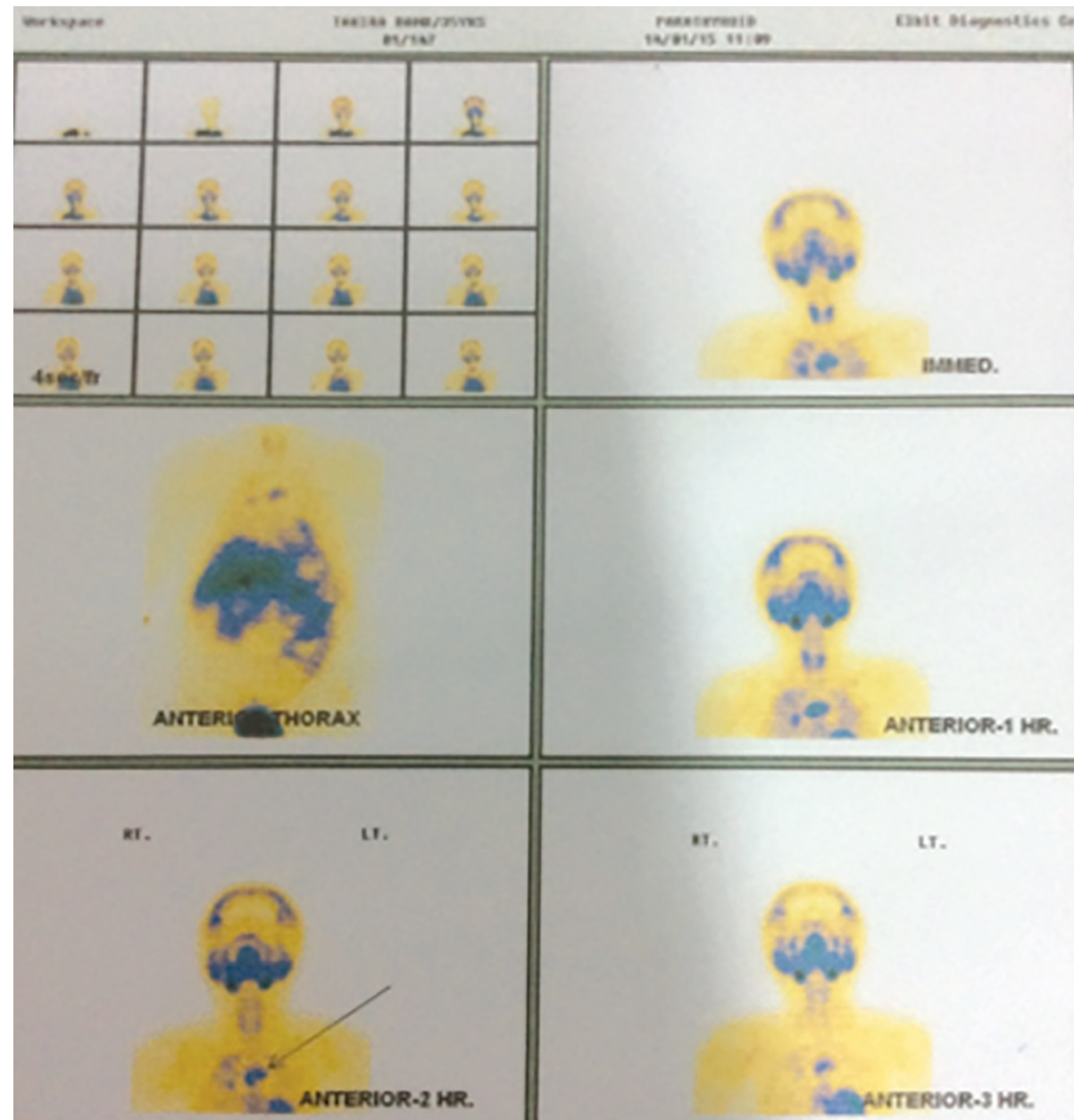

Fig. 3: Technetium-99m sestamibi scan

the aortic arch. An ectopic parathyroid adenoma was localized in the precarinal space on a gallium 68 DOTANOC PET scan.

In view of its location, sternotomy, transpericardial approach, and mediastinal exploration with intraoperative PTH monitoring were planned in collaboration with endocrine and cardiothoracic surgeons. After sternotomy, the thymus, fat, and nodes in the anterior mediastinum were excised. The aortopulmonary space was explored and fat and lymph nodes in it were excised.
The pericardium was opened; the posterior pericardium was approached through dissection of the space between the superior vena cava and the aorta. All the fat including the lymph nodes and a tan colored nodule (parathyroid carcinoma) posterior to ascending aorta and anterior to the tracheal carina were removed. The patient underwent removal of ectopic parathyroid adenoma with an immediate drop in the parathyroid hormone levels (preoperative level $2291.3 \mathrm{pg} / \mathrm{mL}$ to immediate postoperative $604.4 \mathrm{pg} / \mathrm{mL}$ and 


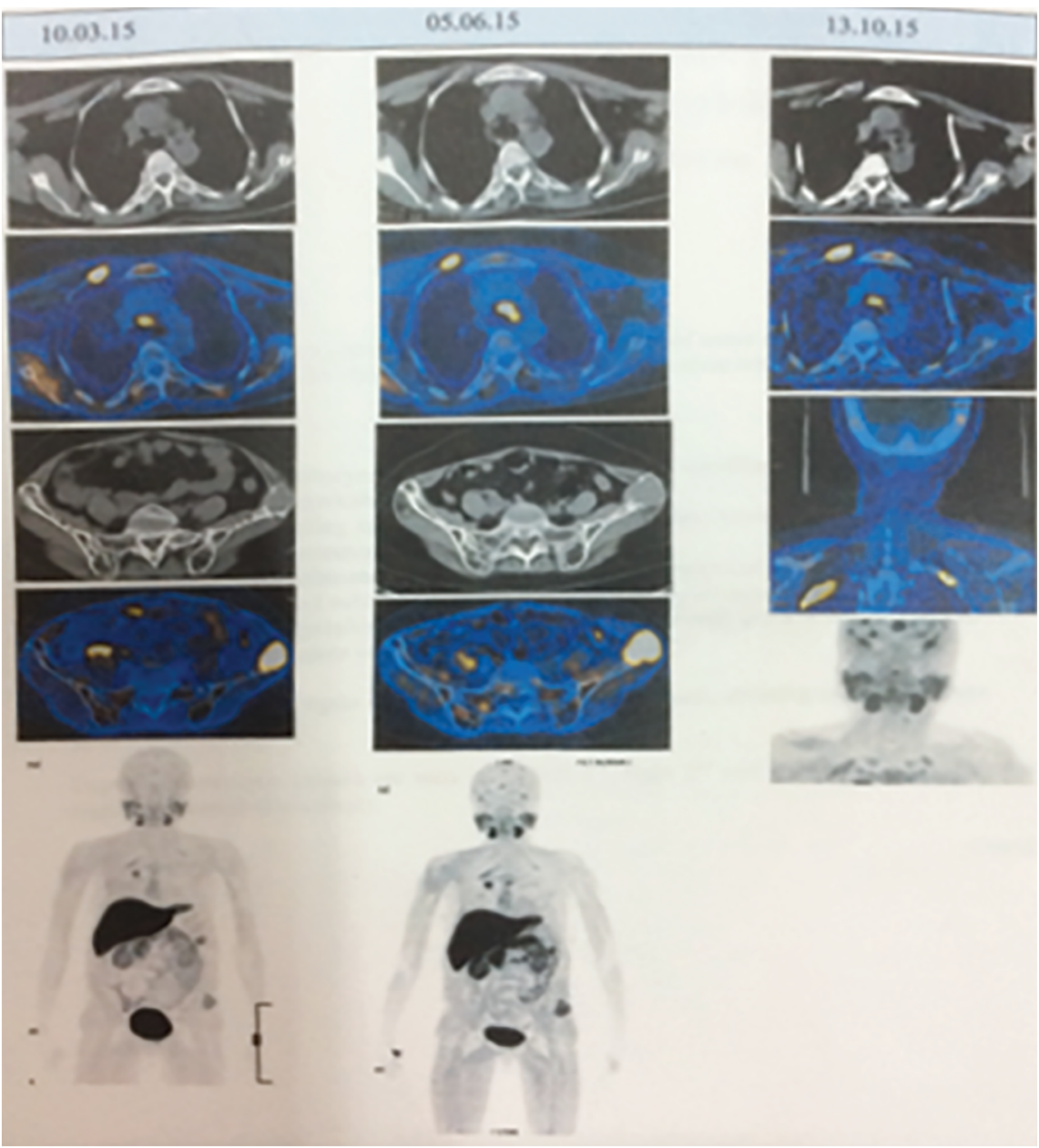

Fig. 4: PET-scan showing anterior mediastinal hot spot

$11.2 \mathrm{pg} / \mathrm{mL}$ on 1st postoperative day) indicating successful surgery. This was an ectopic left inferior parathyroid as it was missing in the neck.

Histopathological report of excised parathyroid gland revealed capsular invasion suggestive of parathyroid carcinoma.

\section{Discussion}

The most common cause of persistent primary hyperparathyroidism is the presence of an ectopic gland. An ectopic parathyroid gland may arise from the superior gland, inferior gland, or supernumerary gland. Most ectopic parathyroid glands located in the mediastinum are found to be supernumerary. Increased use of radionuclide imaging, the advent of selective venous sampling, and intraoperative gamma probe use and 4D-computed tomography scans have all increased the chances of accurate localization, especially after failed neck explorations.

Parathyroid malignancy was first described by de Quervain in 1904, and about one thousand cases of this pathology have been described in the English literature. ${ }^{3}$ Parathyroid carcinoma accounts for $3 \%$ of primary hyperparathyroidism cases and there are no specific guidelines for its staging and management. Chemotherapy and radiation therapy are largely ineffective, and surgery remains the best chance of cure. These tumors are seen typically in the fourth decade but can afflict both sexes equally.
Operative management of patients with persistent hyperparathyroidism is difficult despite the new advances in radiological imaging. Patients are better managed with a combined approach of imaging, selective venous sampling, and intraoperative parathyroid hormone monitoring. ${ }^{4}$ Madou et al. proposed that combining an operative (open transpericardial approach) removal with intraoperative PTH level monitoring gives more accurate result compared with classic "Miami Criteria"-decline by $>50 \%$ from baseline and into the normal range by $10-15$ minutes after resection. ${ }^{5}$ Frozen section is not mandatory but can be used to confirm parathyroid tissue when PTH levels fail to decrease. High cardiac background activity limits the use of technetium-99m methoxyisobutylisonitrile (MIBI) radio-guided surgery in aortopulmonary window parathyroid adenomas. ${ }^{6}$ Appropriate use of preoperative imaging tests and knowledge of the potential location of parathyroid adenomas can lead to very high cure rates with minimal morbidity.

\section{Conclusion}

Parathyroid malignancies are extremely rare but should be considered in patients presenting with clinical and biochemical examinations suggestive of primary hyperparathyroidism. The middle mediastinal location of the ectopic parathyroid gland accounts for $1-5 \%$, and of them, $0.24 \%$ of all are parathyroid adenomas. $^{7}$ The incidence of ectopic parathyroid gland in patients with primary hyperparathyroidism ranges from 9 to $20 \%{ }^{8}$ 
During neck exploration for a hyperfunctioning adenoma, understanding the embryology, and anatomy of the parathyroid glands is essential to achieve surgical cure. Here, we want to emphasize the importance of intraoperative monitoring of PTH levels combined with the surgical transpericardial approach for the removal of ectopic precarinal parathyroid carcinoma.

\section{References}

1. Shah-Patel LR, Ghesani M, Connery C, et al. Gamma probe detection of ectopic parathyroid adenoma. Radiol Case Rep 2008;3(1):161. DOI: 10.2484/rcr.v3i1.161.

2. Mariani G, Gulec SA, Rubello D, et al. Preoperative localization and radioguided parathyroid surgery. J Nucl Med 2003;44(9):1443-1458.

3. De Quervain F. Parastruma maligna aberrata. Deutsche Zeitschr Chir 1904;100:334-352. DOI: 10.1007/BF02819737.

4. Yen TW, Wang TS, Doffek KM, et al. Reoperative parathyroidectomy: an algorithm foe imaging and monitoring of intraoperative parathyroid hormone levels that results in a successful focused approach. Surgery 2008;144(4):611-619; discussion 9-21 10.1016/ j.surg.2008.06.017.

5. Madou ID, Callender GG, Kim AW. Something old, something new: marrying two approaches to resect an ectopic parathyroid adenoma. J Thorac Cardiovasc Surg 2016;151(2):e33-e34. DOI: 10.1016/ j.jtcvs.2015.09.105.

6. Schwarzlmuller T, Brauckhoff K, Løvås K, et al. High cardiac background activity limits $99 \mathrm{~m}$ Tc-MIBI radioguided surgery in aortopulmonary window parathyroid adenomas. BMC Surg 2014;14:22. DOI: 10.1186/1471-2482-14-22.

7. Ali M, Kumpe DA. Embolization of bronchial artery-supplied ectopic parathyroid adenomas located in aortopulmonary window. J Vasc Interv Radiol 2014;25(1):138-143. DOI: 10.1016/j.jvir.2013.10.008.

8. Andrade JS, Mangussi-Gomes JP, Rocha LA, et al. Localization of ectopic and supernumerary parathyroid glands in patients with secondary and tertiary hyperparathyroidism: surgical description and correlation with preoperative ultrasonography and Tc99mSestamibi scintigraphy. Braz J Otorhinolaryngol 2014;80(1):29-34 DOI: $10.5935 / 1808-8694.20140008$ 